\section{Genetic testing for hypertrophic cardiomyopathy: ongoing voyage from exploration to clinical exploitation}

\author{
lacopo Olivotto, ${ }^{1}$ Heba Sh. Kassem, ${ }^{3}$ \\ Francesca Girolami² \\ 'Department of Cardiology, Referral \\ Center for Myocardial Diseases; ${ }^{2}$ Genetic \\ Diagnostics, Careggi University Hospital, \\ Florence, Italy; ${ }^{3}$ Department of \\ Pathology, Clinical Genomics Centre, \\ Faculty of Medicine Alexandria \\ University, and Magdi Yacoub Heart \\ Foundation Serving, Egypt
}

\section{Abstract}

More than two decades have elapsed since the discovery that sarcomere gene defects cause familial hypertrophic cardiomyopathy (HCM). Since then, genetic testing in HCM has developed and expanded, and is now widely available as a potential clinical service in the Western countries. In the meantime, however, the cross-talk between geneticists and clinicians has developed slowly, and still remains unstandardized, with modalities of interaction and degree of mutual comprehension that vary wildly in various settings. In addition, clinicians often question the clinical utility of genetic testing in HCM patients and their families. The apparent lack of practical benefit, in the face of considerable costs, has long hindered large-scale diffusion of genetic testing, particularly in developing countries, and still accounts for understandable (but not always justifiable) resistance on the part of the physicians. However, such resistance is in contrast with considerable evidence supporting a role for molecular diagnosis in tailoring management for HCM patients. We here review several sound clinical reasons in favour of systematic genetic testing in HCM, ranging from identification of complex genotypes, heralding severe disease expression and outcome, to the added benefit of multidisciplinary genetic teamwork, enhancing awareness towards inheritable diseases in the cardiology community. We hope to show that to underestimate the clinical potential of genetic testing in HCM, and to defer its implementation until more advanced knowledge becomes available, is to lose an important opportunity for present improvement in care.

\section{Introduction}

More than two decades have elapsed since the discovery that sarcomere gene defects cause familial hypertrophic cardiomyopathy (HCM). ${ }^{1}$ Since then, genetic testing in HCM has developed and expanded, has become more affordable, and is now largely available, both via private companies and, in European countries, through the national health system. Most panels for HCM genetic screening cover a range of 8 to 15 genes, mostly coding for proteins of the cardiac sarcomere. However, over 20 genes have been described as HCM-causing, and the quest for novel genes is ongoing. ${ }^{2}$ An approach that has proven remunerative is the systematic investigation of candidate genes coding for cardiomyocyte structural proteins, moving along two main directions: the first focuses on genes coding for Z-disc proteins, which are essential for the structural organization of the cardiac sarcomere and the cardiomyocyte's stretch sensor. Genes identified by this approach include titin, muscle LIM protein, telethonin, myozenin 2 and vinculin. The second pathway follows the description of HCM phenocopies caused by mutations in the $\gamma 2$ subunit of AMP-dependent protein kinase (PRKAG2) and in the lysosomal-associated membrane protein 2 (LAMP2) $^{3}$ directing the search towards rare storage syndromes. ${ }^{4}$ The candidate gene approach, however, appears to have been stretched to its limit, and each novel gene identified only accounts for a small fraction of patients in HCM cohorts. In the near future, novel opportunities disclosed by next generation sequencing, including the possibility of a genome-wide approach, will radically change the approach to what is as yet genotype-negative HCM. ${ }^{5}$ Therefore, much has been accomplished and much is still to come in HCM genetics, and the field is growing at an exciting speed.

In the meantime, however, the cross-talk between geneticists and clinicians has developed slowly, and remains unstandardized, with modalities of interaction and degree of mutual comprehension that vary wildly in various settings. Besides sheer problems of communication, which can be easily overcome by regular teamwork, ${ }^{6,7}$ clinicians often question the clinical utility of genetic testing in HCM patients and their families. The apparent lack of practical benefit, in the face of considerable costs, has long hindered large-scale diffusion of genetic testing, particularly in the developing world, and still accounts for understandable (but not always justifiable) resistance on the part of the physicians. Arguably, such approach might have been appropriate in the early phase of HCM genetic testing (i.e. 1990-1999), which can be regarded as Magellan's voyages of discovery in unknown territories. Conversely, the
Correspondence: Iacopo Olivotto, Cardiologia Generale 1, Azienda 0spedaliera Universitaria Careggi, viale Pieraccini 17, 50132, Florence, Italy.

Tel. +39.055.7945138 - Fax: +39.055.7949335.

E-mail: olivottoi@aou-careggi.toscana.it

Key words: hypertrophic cardiomyopathy, genetic testing, phenotype, outcome.

Acknowledgments: this work was supported by Ministero Istruzione Università e Ricerca (PRIN), and European Union (STREP Project 241577 BIG HEART, $7^{\text {th }}$ European Framework Program). The Magdi Yacoub Heart Foundation and Biobliotheca Alexandrina sponsor curren research on genetic testing of hypertrophic cardiomyopathy in Egypt.

Received for publication: 1 June 2011. Accepted for publication: 14 June 2011.

This work is licensed under a Creative Commons Attribution NonCommercial 3.0 License (CC BYNC 3.0).

(C) Copyright I. Olivotto et al., 2011

Licensee PAGEPress, Italy

Cardiogenetics 2011; 1:e3

doi:10.4081/cardiogenetics.2011.e3

following decade (2000-2009) resembles the expeditions of the conquistadores, when newly discovered lands were systematically exploited for riches (Figure 1). Evidence accumulated during this time strongly in supports a clinical role for molecular diagnosis in shaping individualized management for HCM patients. ${ }^{1,7,8}$ In an attempt to entice the sceptical, several sound, practical reasons in favour of systematic genetic testing are discussed below.

\section{Expanding the spectrum of hyper- trophic cardiomyopathy}

The identification of affected family members has been classically invoked by critics as the main, if not only, true benefit of genetic testing, particularly in the presence of mild or equivocal phenotypes. ${ }^{9}$ The introduction of advanced cardiac imaging techniques (first and foremost: cardiac magnetic resonance CMR), has allowed clinicians to identify very subtle abnormalities of the heart that often overlap with the normal..$^{10,11}$ Thus, the dividing line between health and disease has become very thin in individuals with suspected cardiomyopathies. The most striking example of this is certainly arrhythmogeneic right ventricular cardiomyopathy, a condition that may be very difficult to rule in or rule out simply based on CMR findings. ${ }^{12}$

When HCM is suspected, findings such as borderline left ventricular (LV) thickness, areas of LV non-compaction, minor degrees of 
left atrial dilatation, redundant mitral leaflets, may all suggest the presence of a mild phenotype. ${ }^{13}$ However, the only gold standard by which such suspicion can be tested is a positive mutational status for HCM, especially in presence of positive family history. Therefore, in the last decade, large-scale genetic screening performed at referral centres has been key to validating the most subtle findings provided by state-of-the-art imaging, and to expand the HCM disease spectrum towards its milder end. ${ }^{10,11,13}$ Conversely, in HCM families with unavailable or negative genetic test, many individuals remain very difficult to label as affected or non-affected based on clinical assessment alone.

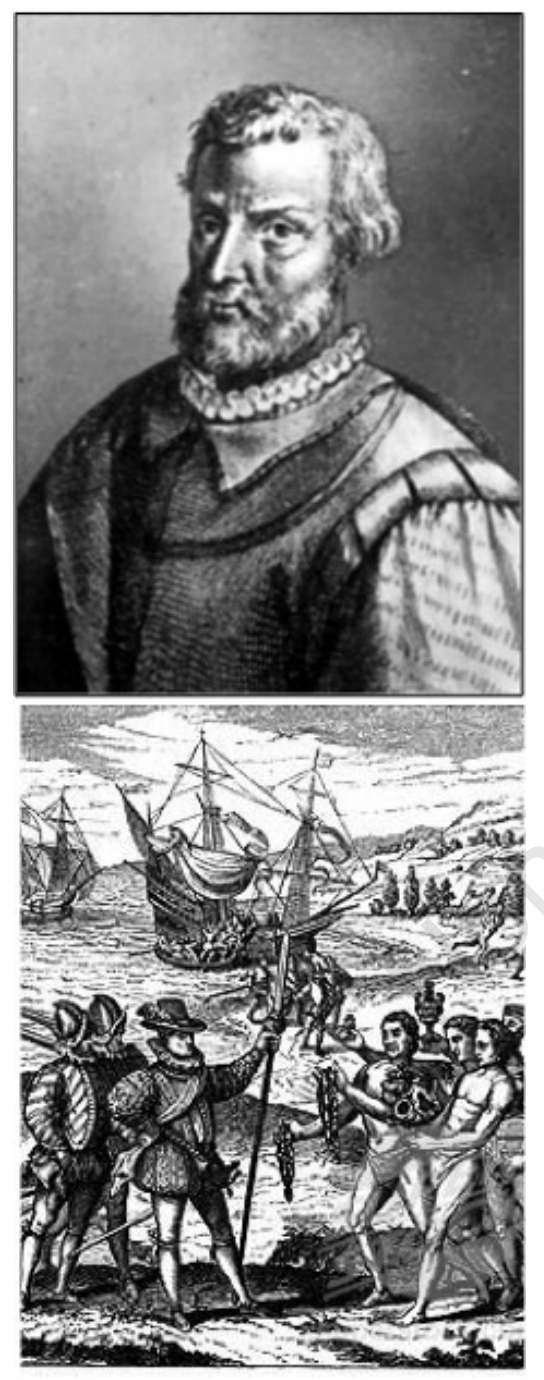

Figure 1. The early phase of hypertrophic cardiomyopathy genetic testing (i.e. before 2000), can be regarded as voyages of discovery in unknown territories, resembling Magellan's (up panel). Conversely, the following decade resembles more the expeditions of the conquistadores (down panel), when newly discovered lands were systematically exploited for riches.
In addition, genetic testing is obviously a sine qua non for the identification of genotype-positive, phenotype-negative individuals, a condition also known as pre-hypertrophic.,14 As previously discussed, the impressive accuracy of current imaging standards is causing a constant shift in HCM phenotype paradigms, so that the definition of truly phenotype-negative individuals is becoming progressively more restrictive. For example, isolated mitral valve and subvalvar abnormalities represent primary expressions of HCM which must be taken into consideration in family members, even in the absence of hypertrophy. ${ }^{15}$ Nevertheless, due to the incomplete penetrance of HCM-causing mutations, a significant subset of genotype-positive individuals will be clinically unaffected and exhibit a truly negative phenotype in any age group. ${ }^{14}$

The clinical history of this peculiar prehypertrophic subset is still largely undefined, but is likely associated with benign clinical course and outcome, even though some of these individuals will develop a clear-cut HCM phenotype over time. ${ }^{2,7}$ In any case, the identification of a genotype-positive status remains important in this subgroup, because of obvious implications for disease transmission to the offspring, deserving appropriate counselling, as well as implementation of follow-up.? Furthermore, young phenotype-negative indi- viduals represent ideal candidates for pharmacological trials aiming at preventing phenotype development, based on encouraging pre-clinical data obtained in transgenic animal models of HCM. ${ }^{1,16}$

An important caveat regards the issue of genetic testing in children from families with HCM. While regular clinical surveillance remains key in this age group, it is our view that minors should not be offered mutational screening in the absence of reasonable clinical evidence of disease. ${ }^{6,17}$ Because the prognostic relevance of a pre-hypertrophic phenotype remains to be determined, it is important that each individual may freely decide for himself/ herself, whether a genotype-positive status should be identified in case of a clinical equivocal diagnosis. In the absence of defined practical advantages (with the only, uncertain and debated exception of reducing risk by restraining sports participation), the burden of anxiety and potential adverse consequences of genetic testing are likely to outweigh benefits in this specific context. ${ }^{17}$ Needless to say, this line of reasoning does not apply to minors in whom a clinical diagnosis of HCM can be made based on standard criteria, either as probands or during family screening. In such occurrence, genetic testing is indicated according to the rules employed for adult patients, pending parental approval. ${ }^{7}$
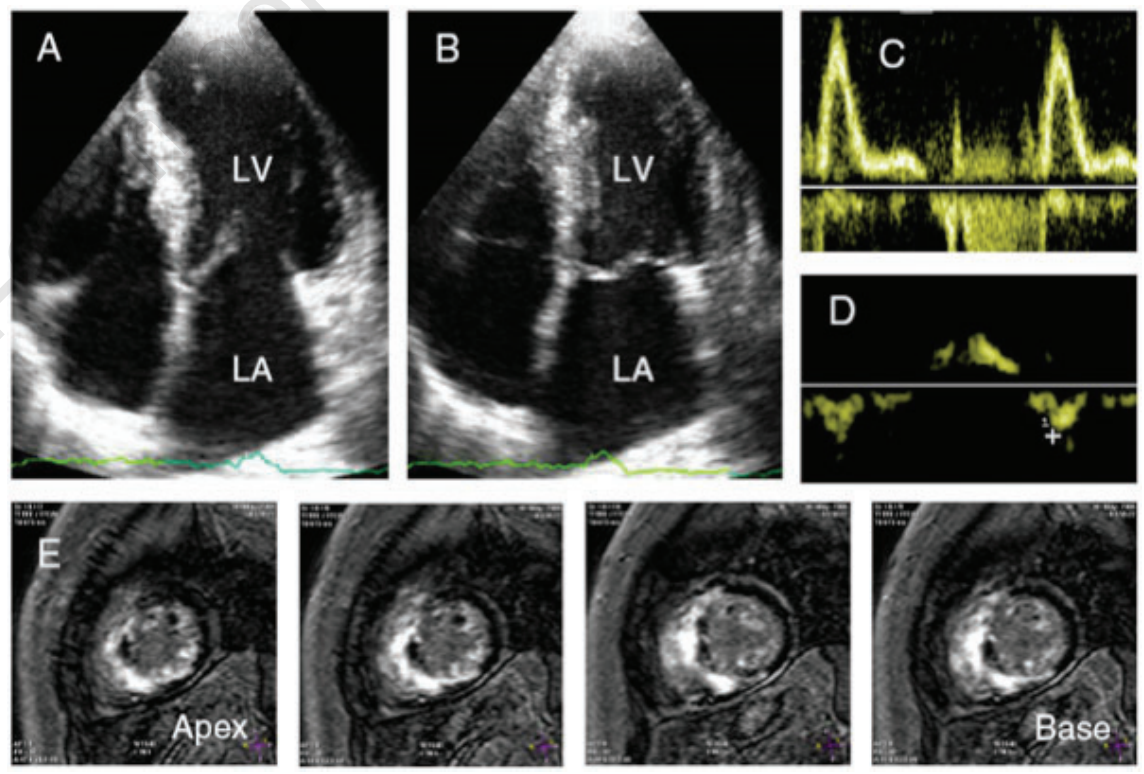

Figure 2. Evidence of severe left ventricular dysfunction and fibrosis in a 32-year old female patient with cardiomyopathy (HCM)caused by triple sarcomere gene defect including a missense mutation in MYH7 $(\mathrm{R869H})$, a splice site mutation in MYBPC3 (E258K), and a frameshift mutation in TNNI3 (A86fs). Echocardiographic apical 4chamber view at end-diastole (A) and end-systole (B) showing mild LV cavity dilation with reduced ejection fraction, a bright, akinetic ventricular septum, and marked left atrial (LA) dilation. Pulsed-wave Doppler showing a restrictive mitral flow pattern (C) associated with severe early diastolic tissue Doppler velocity reduction (D) $E^{\prime} 5 \mathrm{~cm} / \mathrm{s}$. Cardiac magnetic resonance imaging end-diastolic short-axis images demonstrate extensive transmural late gadolinium enhancement in the ventricular septum extending into the posterior free wall (E). Reproduced from ref \#24. 


\section{Sarcomere hypertrophic cardio- myopathy as a progressive disease - the case of complex genotypes}

Early reports promoted the idea that genotyping of HCM patients may prove useful for risk stratification and prediction of long-term outcome. ${ }^{1}$ Subsequent studies, however, have consistently failed to establish meaningful relationships between specific sarcomere myofilament genes, phenotype and outcome. ${ }^{18,19}$ Quite the opposite, the overlap of clinical and pathophysiologic correlates among different genes has shown that genotype-positive, myofilament HCM is a protean but inherently consistent disease, characterized by high prevalence of familial trait and substantial progression towards heart-failure related complications. $^{20}$ In this respect, genotype-negative disease appears to behave differently, in that a familial trait is less prevalent, and the longterm course significantly more stable and benign. ${ }^{1,20}$

Patients with complex genotypes represent an important case in point supporting the concept of myofilament HCM as a progressive disease. A number of independent studies consistently show that multiple sarcomere defects, independent of whether affecting the same or different genes, are associated with earlier onset and more severe clinical phenotype and disease course. ${ }^{21-23}$ In a recent paper describing four HCM patients with triple myofilament gene mutations, representing rare and extreme paradigms of complex genotype, two had a history of resuscitated cardiac arrest or appropriate defibrillator intervention, and three progressed to end-stage HCM by the fourth decade, requiring cardiac biventricular pacing or transplantation ${ }^{24}$ (Figure 2). These adverse consequences likely reflect more profound derangement of sarcomere mechanics and cardiomyocyte energetics caused by multiple mutations, ${ }^{25}$ but may also be mediated by greater impairment of coronary microvascular function, selectively caused by sarcomere gene defects via unknown molecular pathways. ${ }^{26}$

In the specific HCM patient subsets, genetic screening may thus, provide important additional clues to risk stratification, and potentially indicate the need for differential surveillance strategies based on genotype. Specifically, patients with multiple mutations should be considered for close clinical and imaging follow-up, in order to allow timely recognition of disease progression and increased arrhythmic risk..$^{24}$ In the presence of an initial decline in systolic function, the initiation of ACE-inhibition or angiotensin receptor blockade might prevent further LV remodelling and overt systolic dysfunction. ${ }^{13,20,24}$ Furthermore, patients with complex genotypes may require heightened attention with regard to primary prophylaxis of sudden cardiac death. ${ }^{24}$

\section{The issue of pre-natal diagnosis}

In individual HCM families with particularly severe outcome, mostly related to multiple juvenile sudden deaths, prenatal diagnosis has been successfully employed to terminate pregnancies in which the foetus carried the disease-causing mutation. ${ }^{27}$ Nevertheless, because of the uncertain genotype-phenotype relationship, as well as the unpredictable, often benign course of HCM, such an aggressive approach should be considered only in extremely selected instances.

\section{Re-thinking the diagnosis in geno- type-negative patients}

To date, genotype-negative HCM represents a composite entity, probably comprising a multitude of rare, heterogeneous, and yet-to-be identified HCM-susceptibility genes, as well as, potentially, sporadic disease of non-genetic aetiology. ${ }^{1,318-20}$ In clinical practice, each patient with unequivocal HCM phenotype and negative first-line genetic screening deserves in-depth individual assessment of alternative molecular diagnoses, including rare HCM-

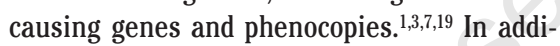
tion, particularly in the young, a negative mutational screening for canonical HCM genes should prompt the search for subtle extra-cardiac manifestations, including abnormal facial and body features and minor renal impairment or neurological deficits. These signs, often overlooked by cardiologists, might point diagnostic efforts in the right direction, highlighting the importance of multidisciplinary approach involving clinical geneticists. ${ }^{7}$

\section{The added value of genetic coun- selling in clinical practice}

An indisputable, although not always appreciated benefit of systematic genetic testing in HCM lies in the cross-fertilization between cardiologists and geneticists. The former, generally show limited expertise and propensity at investigating the hereditary nature of cardiac diseases, and at identifying complex, syndromic phenotypes associated with cardiomyopathies (such as Noonan's, Leopard's, mitochondrial disease and Anderson Fabry, just to name a few). ${ }^{1,7,18,19,28}$ Standard protocols for HCM genetic testing routinely include pre-test counselling by a multidisciplinary team involving clinical geneticists (Figure 3). This is a valuable moment for reciprocal education among professionals, ultimately benefitting a wide spectrum of patients with rare conditions. Of note, diseases ranging from idiopathic $\mathrm{LV}$ dysfunction to bicuspid aortic valve may have a clear familial background that is all too often neglected in the course of routine clinical evaluations. Implementing systematic protocols for genetic screening of a single entity, in our case HCM, generally proves an impor-

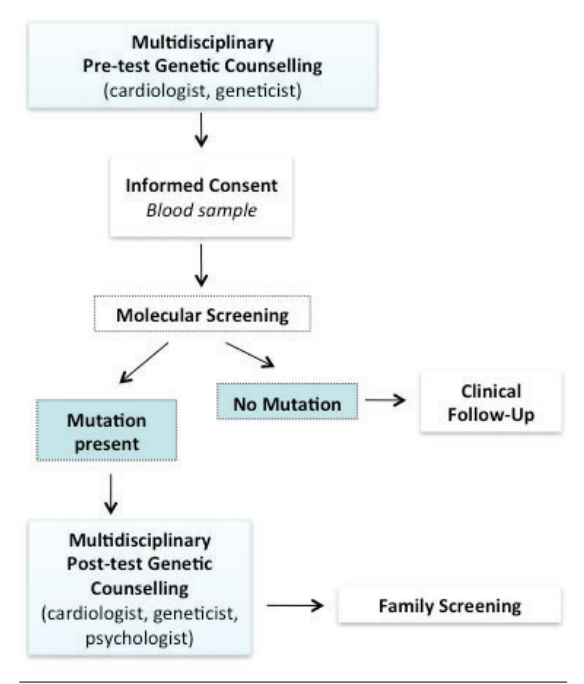

Figure 3. Diagram illustrating the multidisciplinary protocol followed at our Institutions for counseling and administration of genetic testing in inherited cardiomyopathies.

tant step towards improved recognition and management of inherited heart disorders at large. $1,18,19,29$

\section{Conclusions}

Genetic testing for HCM cannot be regarded as an expensive academic gadget, but rather represents a valuable tool in the clinical armamentarium. While major questions remain unanswered, calling for renovated efforts in translational research, what has been achieved so far is sufficient to establish the role of genetic testing in tailored management of HCM patients and their families. To underestimate the actual potential of genetic testing in HCM, and to defer its implementation until more advanced knowledge is available, is to lose an important opportunity for present improvement in care.

\section{References}

1. Watkins H, Ashrafian H, Redwood C. Inherited Cardiomyopathies. N Engl J Med 2011; 364:1643-56.

2. Ho CY. Genetics and clinical destiny: improving care in hypertrophic cardiomyopathy. Circulation 2010;122:2430-40.

3. Arad M, Maron BJ, Gorham JM, et al. Glycogen storage diseases presenting as hypertrophic cardiomyopathy. N Engl J Med 2005;352:362-72.

4. Ahmad F, Arad M, Musi N, et al. Increased alpha2 subunit-associated AMPK activity and PRKAG2 cardiomyopathy. Circulation 
2005;112:3140-8.

5. Wheeler M, Pavlovic A, DeGoma E, et al. A new era in clinical genetic testing for hypertrophic cardiomyopathy. Cardiovasc Transl Res 2009;2:381-91.

6. Girolami F, Olivotto I, Passerini I, et al. A molecular screening strategy based on beta-myosin heavy chain, cardiac myosin binding protein $\mathrm{C}$ and troponin $\mathrm{T}$ genes in Italian patients with hypertrophic cardiomyopathy. J Cardiovasc Med (Hagerstown) 2006;7:601-7.

7. Charron P, Arad M, Arbustini E, et al. Genetic counselling and testing in cardiomyopathies: a position statement of the European Society of Cardiology Working Group on Myocardial and Pericardial Diseases. Eur Heart J 2010;31:2715-26.

8. Seidman CE, Seidman JG. Identifying sarcomere gene mutations in hypertrophic cardiomyopathy: a personal history. Circ Res 2011;108:743-50.

9. Maron BJ, Yeates L, Semsarian C. Clinical challenges of genotype positive (+)-phenotype negative (-) family members in hypertrophic cardiomyopathy. Am J Cardiol 2011;107:604-8.

10. Nagueh SF, Bierig SM, Budoff MJ, et al. American Society of Echocardiography clinical recommendations for multimodality cardiovascular imaging of patients with hypertrophic cardiomyopathy: Endorsed by the American Society of Nuclear Cardiology, Society for Cardiovascular Magnetic Resonance, and Society of Cardiovascular Computed Tomography. J Am Soc Echocardiogr 2011;24:473-98.

11. Maron MS, Maron BJ, Harrigan C, et al. Hypertrophic cardiomyopathy phenotype revisited after 50 years with cardiovascular magnetic resonance. J Am Coll Cardiol 2009;54:220-8.

12. Vermes E, Strohm 0, Otmani A, et al. Impact of the revision of arrhythmogenic right ventricular cardiomyopathy/dysplasia task force criteria on its prevalence by CMR criteria. JACC Cardiovasc Imaging 2011;4:282-7.

13. Olivotto I, Girolami F, Nistri S, et al. The many faces of hypertrophic cardiomyopathy: from developmental biology to clinical practice. J Cardiovasc Transl Res 2009;2:349-67.

14. Ho CY, López B, Coelho-Filho OR, et al. Myocardial fibrosis as an early manifestation of hypertrophic cardiomyopathy. $\mathrm{N}$ Engl J Med 2010;363:552-63.

15. Maron MS, Olivotto I, Harrigan C, et al. Mitral valve abnormalities identified by cardiovascular magnetic resonance represent a primary phenotypic expression of Hypertrophic Cardiomyopathy. Circulation 2011 Jun 13. [Epub ahead of print]

16. Senthil V, Chen SN, Tsybouleva N, et al. Prevention of cardiac hypertrophy by atorvastatin in a transgenic rabbit model of human hypertrophic cardiomyopathy. Circ Res 2005;97:285-92.

17. Borry P, Evers-Kiebooms G, Cornel MC, et al. Genetic testing in asymptomatic minors: background considerations towards ESHG Recommendations. Eur J Hum genet 2009;17:711-9.

18. Wang L, Seidman JG, Seidman CE. Narrative review: harnessing molecular genetics for the diagnosis and management of hypertrophic cardiomyopathy. Ann Intern Med 2010;152:513-20.

19. Hershberger RE, Lindenfeld J, Mestroni L, et al. Genetic evaluation of cardiomyopathy--a Heart Failure Society of America practice guideline. J Card Fail 2009;15:8397.

20. Olivotto I, Girolami F, Ackerman MJ, et al. Myofilament protein gene mutation screening and outcome of patients with hypertrophic cardiomyopathy. Mayo Clin Proc 2008;83:630-8.

21. Van Driest SL, Vasile VC, Ommen SR, et al. Myosin binding protein $\mathrm{C}$ mutations and compound heterozigosity in hypertrophic cardiomyopathy. J Am Coll Cardiol 2004; 44:1903-10.

22. Ingles J, Doolan A, Chiu C, et al. Compound and double mutations in patient with hypertrophic cardiomyopathy: implications for genetic testing and counselling. J Med Genet 2005;42:e59.

23. Kelly M, Semsarian C. Multiple mutations in genetic cardiovascular disease; a marker of disease severity. Circ Cardiovasc Genet 2009;2:182-90.

24. Girolami F, Ho CY, Semsarian C, et al. Clinical features and outcome of hypertrophic cardiomyopathy associated with triple sarcomere protein gene mutations. J Am Coll Cardiol 2010;55:1444-53.

25. Belus A, Piroddi N, Scellini B, et al. The familial hypertrophic cardiomyopathyassociated myosin mutation $\mathrm{R} 403 \mathrm{Q}$ accelerates tension generation and relaxation of human cardiac myofibrils. J Physiol 2008;586;3639-44.

26. Olivotto I, Girolami F, Sciagrà R, et al. Microvascular function is selectively impaired in hypertrophic cardiomyopathy patients with sarcomere myofilament gene mutations. Medicine 2010 [Epub ahead of print].

27. Charron P, Héron D, Gargiulo M, et al. Prenatal molecular diagnosis in hypertrophic cardiomyopathy: report of the first case. Prenat Diagn 2004;24:701-3.

28. van Langen IM, Birnie E, Leschot NJ, et al. Genetic knowledge and counselling skills of Dutch cardiologists: sufficient for the genomics era? Eur Heart J 2003;24:560-6.

29. Christiaans I, van Langen IM, Birnie E, et al. Genetic counseling and cardiac care in predictively tested hypertrophic cardiomyopathy mutation carriers: the patients' perspective. Am J Med Genet A 2009;149A: 1444-51. 